\title{
An Exploring Research on Key Technology of CAN Bus Configuration for the Industrial Automation Applications
}

\author{
Xuexuan $\mathrm{ZHU}{ }^{1, a}$ \\ ${ }^{1}$ College of Electrical and Electronic Engineering, Xin Yu University, Xin Yu 338004, China \\ axuexuanzhu@126.com
}

Keywords: Industrial Automation; CAN Bus; Configuration Software

\begin{abstract}
Herein fieldbus control system configuration software is hardware platform, the first about the definition and development of domestic and international fieldbus, specifically discusses the characteristics and hierarchical structure CAN bus, followed by a detailed analysis of the development of the configuration software situation, proposed the establishment of the hardware platform of CAN bus control system. Fieldbus control system is the development direction of computer control system, the field bus technology to the industrial control system, the paper CAN bus configuration key technologies of industrial automation applications in-depth research, can lay the groundwork for the eventual integration of monitoring and control.
\end{abstract}

\section{Introduction}

Development of computer control systems, industrial control so increased complexity, the number increases, the industry has begun to standardize control systems, industrial control systems can constitute a combination of hardware equipment has been formed, which makes industrial control systems compatibility and reliable, interchangeability have greatly increased, and makes the system easy to upgrade and replace [1-2]. The industrial control system software development it is relatively backward, which makes most users and contractors need to develop their own appropriate application software, which not only extended the development cycle, and because of the uneven development level, so that the application level is not high poor quality, resulting in a large number of low-level of unnecessary duplication. It is recognized that effective industrial control system software development tools in industrial control system software development process is essential. Thus the emergence of a new type of industrial control configuration software, the user can control the configuration software industry, they constitute themselves through the configuration process required for industrial control system software [3].

CAN (Controller Area Network) bus data exchange is to address the numerous car between control and test equipment and the development of a serial data communication protocol, which is a multi-master bus, the communication medium can be twisted pair, coaxial cable or optical fiber. Because CAN outstanding features, high reliability and unique design, especially for the interconnection of industrial process monitoring equipment, so the industry more and more attention, and International Organization for Standardization has officially promulgated CAN international standards (ISO11898), CAN bus has been recognized as one of the most promising field bus [4]. So in the long term, highly developed fieldbus system will improve the level of automation and control industrial field. Therefore, the CAN bus used in industrial field is a good development, to improve the industrial field automation control level, to strengthen industrial control accuracy, timeliness and effectiveness, play a decisive role, and enhancing our automation technology the overall level of great significance to further accelerate China's industrial automation process.

\section{CAN bus technology}

Fieldbus [gate is connected intelligent field devices and automation systems for digital, two-way transmission, multi-branch structure of the communication network. It is the computer technology, communication technology and control technology is highly integrated with the integrated product, 
is an open and distributed River. The new mode is the focus of today's fieldbus automation technology development, computer local area is known as the field of automation layered .CAN bus network [5]. It is more and more widely used in industrial control, intelligent buildings and transportation and many other areas to follow ISO / OSI standard model, CAN into the data link layer (including the LLC sub-layer LLC and MAC sub-layer MAC) and physical layer, and in the canonical version 2.0A CAN technology, the services and functions of the LLC data link layer and the MAC sub-layer is described as the 'target layer' and 'Transport Layer'. CAN hierarchical structure and functions shown in Figure 1.

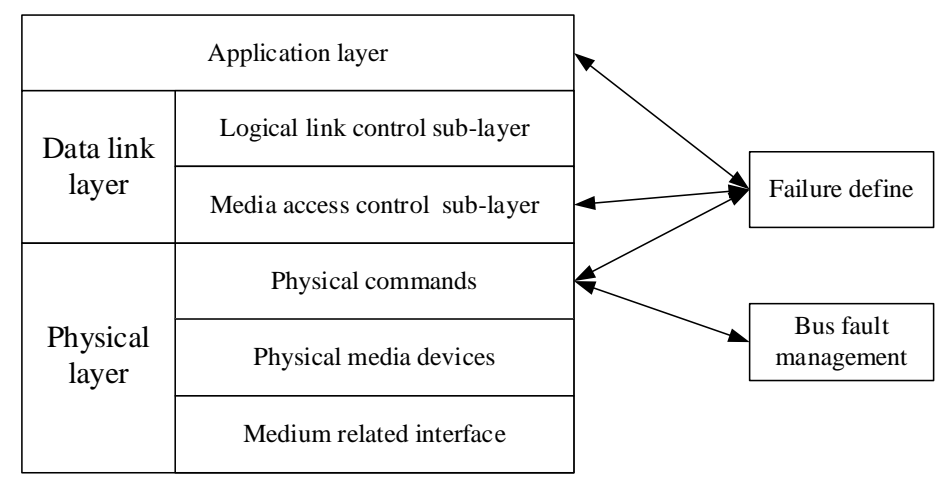

Figure 1.The hierarchical structure of CAN bus

Fieldbus as a digital communications network has been extended to the production site in field equipment, used in the past for individual point type parallel transmission of analog or digital signal transmission signal becomes multi-point bidirectional serial digital line transmission [6-8]. The essential meaning of the field bus performance in the following areas:

(1) Field device connectivity. Site equipment means production site located sensors, transmitters and actuators. These field devices can be interconnected in the field directly through the fieldbus, mutual exchange of information. In the DCS system, it cannot be directly exchanged between field device information.

(2) Interoperability. A wide range of field devices, a manufacturer may not provide all the equipment in an industrial production process needs. In addition, users do not want to subject to a certain manufacturer, so it is possible in a fieldbus control system, connecting multiple manufacturer of equipment. Interoperability refers to the so-called devices from different manufacturers can communicate with each other, it is reflected in: the user can freely select the device, and this option vendor-independent, control systems and communication protocols; manufacturers have to add new, useful ability to function; no proprietary protocols and special custom driver software and software upgrades.

(3) Dispersion function blocks. Fieldbus control system transfer function blocks distributed to field instruments executed, thereby eliminating the traditional DCS systems control the process control station. For example, fieldbus transmitters will have in addition to the general functions of the transmitter can also run PID control function blocks. Similarly, the fieldbus actuators addition to the general actuator functions, it can also run PID control function block and output characteristics compensation block can even achieve the valve characteristic self-checking and self-diagnostic function valve.

(4) Fieldbus power supply Fieldbus addition to transmitting information, but also can be done as a function of field devices. Bus-powered wiring system not only simplifies installation, but also the nature of the safety gate system can be achieved by supporting the barrier, the fieldbus control system applications in explosive environments foundation.

(5) Open fieldbus network is an open network, both with the same level networking, but also with different layers of network interconnection. Fieldbus protocol is a fully open protocol, unlike DCS as use of closed, proprietary communications protocols, instead of using open, standardized communication protocols. This means that the fieldbus devices from different manufacturers, provided that they meet the fieldbus protocol, you can connect to the system via a fieldbus network, integrated automation. 


\section{Fieldbus distributed control of CAN}

Currently, the configuration software in the control system based on CAN bus and rare. General CAN bus PC monitoring software development mainly in two ways: First, developed separately PC software, to achieve the lower computer communications, but is bound to result in duplication of work; the second is to use a dedicated connection tool, the CAN bus and commercialization The configuration software is connected, but the commercial configuration software is expensive, for some SMEs, and university teaching is difficult to accept, and the actual needs of enterprises there is a large distance. Therefore, the development of the protocol is simple, reliable communications configuration software CAN-bus control system is very necessary. Distributed control system based on CAN field bus structure is shown in figure 2 .

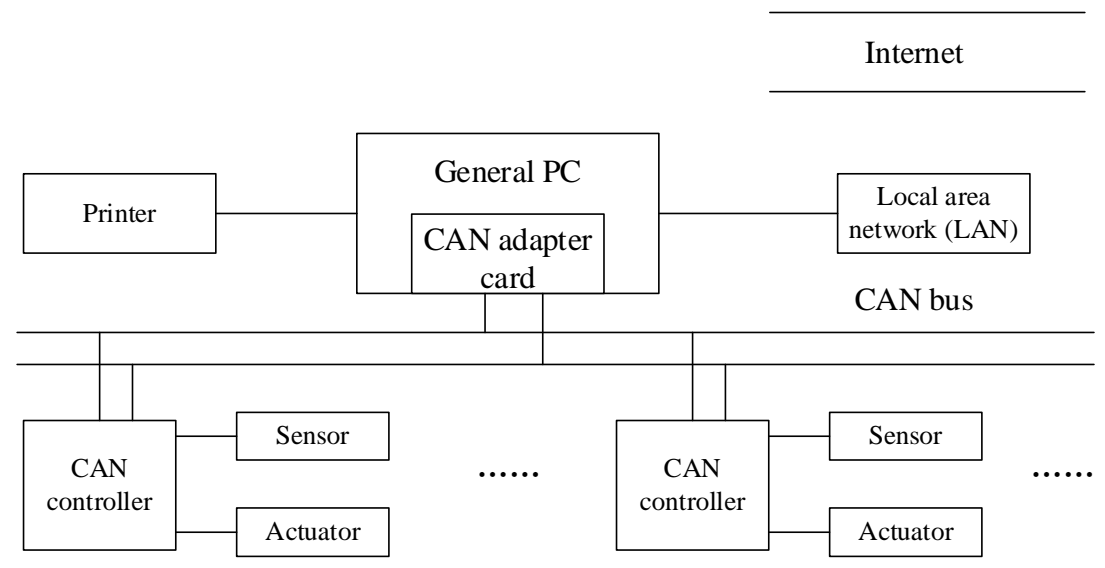

Figure 2.Type of fieldbus distributed control of CAN

This study for industrial automation applications configuration software is built on a hardware platform over CAN bus control system. First, based on research on configuration of software architecture, modular design method to achieve the basic functions of the configuration software framework, established the overall design configuration software, configuration software enables real-time database, graphical configuration and I / O-driven functionality. Then, specific research and design of the modules. In the real-time database to study the data object model, data storage, integration with Windows systems, ADO technical configuration of the database; use of multi-threading technology to improve the efficiency of the program in the graphics configuration, making the system more friendly on the interaction; in I/O drivers, mainly using dynamic link library technology to achieve, in the driver underlying communication programming, implementation using MS control to complete serial communication. Finally, the present configuration software applied to the experimental teaching CAN bus, CAN bus control system makes networking easier and more intuitive control, simple. The configuration software is effective in reducing the engineering staff to develop PC software duplication of work and improve the efficiency of its engineering development; and this applies to CAN bus configuration software Experimental Teaching in Universities. While the CAN bus technology configuration software development has a certain role in promoting research and implement other bus configuration software also has some reference to the role.

\section{CAN bus configuration structure for industrial automation applications}

Research on the contents of the system is the FCS control system based on configuration software development, to build the underlying database, and lower communication and achieve a good man-machine interface. The system is applied to the host computer user to control the display interface, database development and implementation of upper and lower computer communication underlying the completion of data acquisition, signal conversion processing, graphics, data communications, control, report printing and other functions, as shown in Figure 3. 


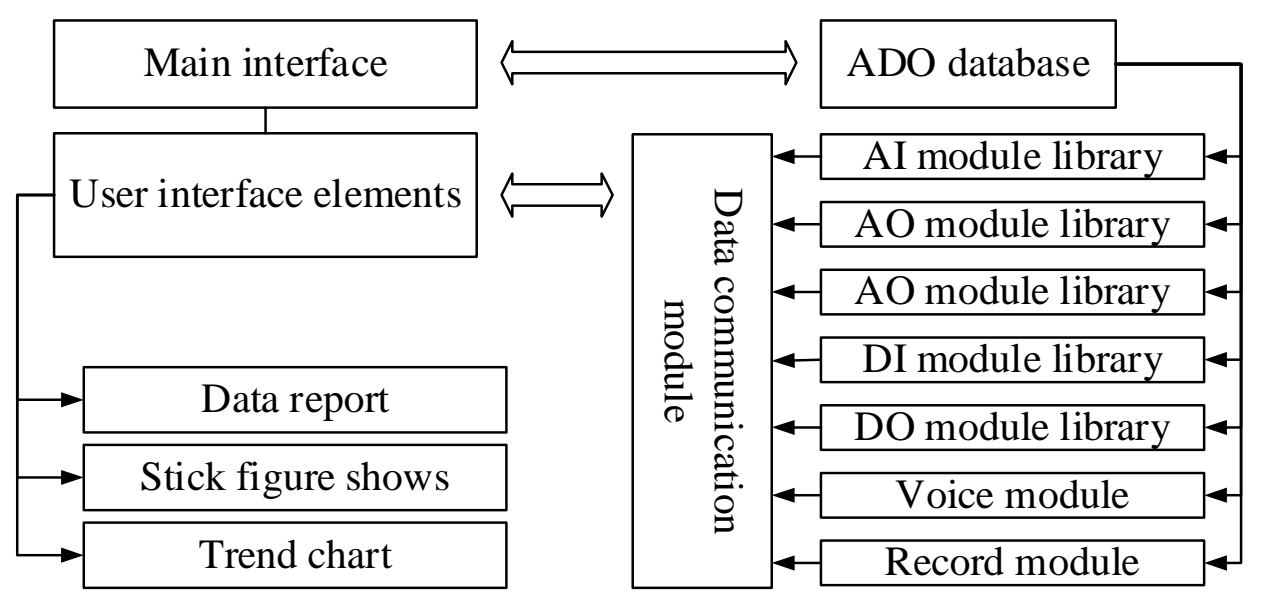

Figure 3. CAN bus configuration structure for industrial automation applications

(1) Configuration interface functions. Configuring the system's user interface with standard Windows graphics applications basic functionality. For loop configuration, the system is set up to produce a configuration file, view the configuration file, and compiling the configuration file, downloading the configuration file and print configuration files and other functions.

(2) On-line configuration capabilities. The whole system is open, users may need to add their own function blocks, namely adding the corresponding information in the function block library. So you can join in the system of advanced control algorithms and neural networks, predictive control, fuzzy control; the way the system configuration is independent of any one specific control system.

(3) Real-time multi-tasking capabilities. Real-time generally require a computer with multi-tasking capabilities, so that monitoring and control tasks into several parallel execution of multiple tasks, accelerate the program execution speed; those changes may not be significant, even if not immediately react not have an impact incident or damage as a sequence of tasks performed in accordance with certain inspection cycle regularly perform.

(4) Good opening. Socialized mass production, so all the hardware and software system cannot be constructed by a company's products, "heterogeneous" is one of the main features of today's control systems; open refers to the configuration software with a variety of Internet protocols, supports a variety of hardware devices, openness is a measure of the quality indicators of a configuration software.

(5) Rich functionality modules. Provide rich control function libraries, and control to meet user requirements and site requirements. Using a variety of functional modules, complete real-time monitoring, generating real-time reports, historical curve display, real-time curve, provide alarm functions, the system has good man-machine interface, easy to operate.

(6)Powerful database. With real-time database, you can store a variety of data, such as analog, discrete, character, etc., to achieve data exchange with external devices. Improve real-time systems, enhanced processing power.

\section{Conclusion}

With the development of distributed computer control systems, there is growing emphasis on software configuration and configuration control system, the system is configured to generate a fully functional configuration tool. Configuration software very versatile, can be applied to a large class of common objects, and execute the program code of the system are generally fixed, to change to a different application objects simply by changing the data entities, data entities, including graphic files, Reports documents and control loop files. CAN bus for industrial automation that is in the system hardware configuration and system software will be the basis of the functional mode system software configuration blocks are connected together in order to achieve the purpose of controlling the process. 


\section{Reference}

[1] Gunasekaran A, Ngai E W T. The future of operations management: an outlook and analysis[J]. International Journal of Production Economics, 2012, 135(2): 687-701.

[2] Baldwin R, Venables A J. Spiders and snakes: offshoring and agglomeration in the global economy[J]. Journal of International Economics, 2013, 90(2): 245-254.

[3] Stone-Gross B, Abman R, Kemmerer R A, et al. The underground economy of fake antivirus software[M]//Economics of Information Security and Privacy III. Springer New York, 2013: 55-78.

[4] Swanson E T, Reichlin L, Wright J H. Let's Twist Again: A High-Frequency Event-Study Analysis of Operation Twist and Its Implications for QE2 [with Comments and Discussion][J]. Brookings Papers on Economic Activity, 2011: 151-207.

[5] Liu Z, Huang M, Yi X. Optimal energy configuration analysis of micro grid based on reliability and economy[C]//Information Science, Electronics and Electrical Engineering (ISEEE), 2014 International Conference on. IEEE, 2014, 1: 326-330.

[6] Gallouj F Ï, Weber K M, Stare M, et al. The futures of the service economy in Europe: a foresight analysis[J]. Technological Forecasting and Social Change, 2015, 94: 80-96. 